\title{
Impact of Multipath Reflections on Secrecy in Visible Light Communication Systems with Randomly Located Eavesdroppers
}

\author{
Sunghwan Cho, Gaojie Chen, Hyunchae Chun, Justin P. Coon, and Dominic O'Brien \\ Department of Engineering Science, \\ University of Oxford, OX1 3PJ, United Kingdom. \\ Email: \{sunghwan.cho, gaojie.chen, hyunchae.chun, justin.coon and dominic.obrien\}@eng.ox.ac.uk
}

\begin{abstract}
Considering reflected light in physical layer security (PLS) is very important because a small portion of reflected light enables an eavesdropper (ED) to acquire legitimate information. Moreover, it would be a practical strategy for an ED to be located at an outer area of the room, where the reflection light is strong, in order to escape the vigilance of a legitimate user. Therefore, in this paper, we investigate the impact of multipath reflections on PLS in visible light communication in the presence of randomly located eavesdroppers. We apply spatial point processes to characterize randomly distributed EDs. The generalized error in signal-to-noise ratio that occurs when reflections are ignored is defined as a function of the distance between the receiver and the wall. We use this error for quantifying the domain of interest that needs to be considered from the secrecy viewpoint. Furthermore, we investigate how the reflection affects the secrecy outage probability (SOP). It is shown that the effect of the reflection on the SOP can be removed by adjusting the light emitting diode configuration. Monte Carlo simulations and numerical results are given to verify our analysis.
\end{abstract}

Index Terms-Physical layer security, visible light communication, reflection, stochastic geometry, secrecy outage probability.

\section{INTRODUCTION}

The past few decades have witnessed the acceleration in development of groundbreaking information technology applications, such as smart phones/tablets, Internet of Things (IoT) devices, virtual reality devices and so on. These new applications require a significant amount of data traffic to be conveyed wirelessly. Furthermore, the growing number of devices imposes a big challenge of providing seamless and low-delay communication [1], [2]. To satisfy these requirements, the future wireless network calls for a new air interface and/or more spectrum, since traditional radio frequency (RF) communication systems have arrived at a bottleneck due to limited spectrum resources. Among many possible mediums, visible light has gained great popularity among researchers as a possible solution for future indoor wireless communications. Visible light communications (VLC) utilize the visible light spectrum from $400 \mathrm{THz}$ to $700 \mathrm{THz}$. This spectrum is license free and can provide a wide bandwidth. Hence, it can be utilized it for high-speed indoor wireless communication [3].

At the same time, in VLC systems, network security remains an important challenge, which needs to be studied further. In large rooms such as offices, libraries, and shopping malls, there is always the possibility that an eavesdropper (ED) can wiretap the message over the air, because any other device in such open spaces can observe the visible light signal. As one of many network security approaches, physical layer security (PLS) is a set of techniques that enables a transmitter and a legitimate receiver to securely communicate important data, eliminating the possibility of eavesdropping by utilizing the randomness of the channel between the transmitter and the receiver [4], [5]. There already exists work on PLS in VLC systems in [6][10]. Lampe et al. analyzed the achievable secrecy rate for single-input single-output (SISO) and multiple-input singleoutput (MISO) scenarios, and proposed various beamforming and jamming schemes to enhance the confidentiality of VLC links [6]-[8]. In addition, Alouini et al. proposed the truncated normal input distribution and the truncated generalized normal input distribution to increase the secrecy capacity under the amplitude constraints of the input signal [9], [10]. However, most of this work assumes that the transmitter knows the locations or channel state information (CSI) of EDs. Yet, it may not be feasible to obtain this information when malicious users are present in the environment. To address this issue, [11] developed a stochastic geometric model to characterize EDs and analyzed the secrecy outage probability (SOP) in VLC systems in the presence of randomly distributed EDs.

Inspired by the previous contributions exploiting stochastic geometry in VLC systems, in this paper, we investigate the impact of the visible light signal reflections on secrecy performance when EDs are randomly distributed. The diffused paths in VLC systems have been neglected in previous PLS works. However, as shown in [12], an ED can acquire legitimate information by tapping a small portion of the reflected light leaked out from a small gap under a door. Moreover, from the EDs perspective, a clever wiretapping strategy would be to intercept the reflected signal near the walls of a room in order to escape the vigilance of a legitimate user (UE). To the best of our knowledge, this is the first paper to consider the reflected light in the context of PLS in VLC systems with random EDs. The contributions of this paper can be summarized as follows:

- we investigate the impact of reflections on the received optical power, and quantify the generalized error in SNR that occurs when reflections are ignored.

- we analyze how the reflected light affects the SOP 


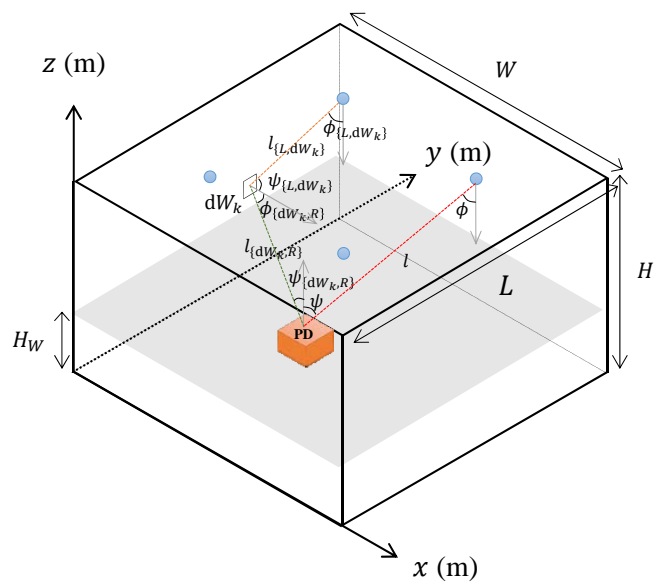

Fig. 1. Propagation model of the LoS and the diffused link. $W$ and $L$ are the room's width and length, and $H$ and $H_{W}$ denote the height of the ceiling and the height from the ceiling to the work plane, respectively. Dots denote LED transmitters.

according to the location of the UE and the configuration of light emitting diode (LED) transmitters.

The rest of this paper is organized as follows. Section II begins with the system model describing the modulation and transmission schemes and providing the performance measures. In Section III, the domain of interest based on the generalized error is investigated. Section IV discusses the impact of the reflected light on the SOP. Section V provides results that support our analysis. Section VI concludes the paper.

\section{SYSTEM MODEL}

\section{A. Room Configuration}

We consider a cuboid room as shown in Fig. $1 . W$ and $L$ denote its width and length, respectively, and $H$ denotes its height. We assume that multiple LED transmitters ${ }^{1}$ are attached to the ceiling, and all photodiode (PD) receivers are located on the work plane (the shaded plane) located $H_{w}$ above from the floor. Also, we assume that there is one UE, and multiple non-colluding EDs are randomly distributed by a Poisson point process (PPP) $\Phi_{E}$ with the density $\lambda_{E}$.

\section{B. Data Transmission and Channel Model}

We consider the DC-biased pulse-amplitude modulation (PAM) VLC scheme as in [8]. The data signal $s(t) \in \mathbb{R}$ is superimposed on a fixed bias current $I_{D C} \in \mathbb{R}^{+}$, where $\mathbb{R}$ and $\mathbb{R}^{+}$denote the set of real-valued numbers and nonnegative real-valued numbers, respectively. This fixed current is used for the function of illumination. The modulated signal $x(t ; \alpha)$ of $s(t)$ can be expressed by $x(t ; \alpha)=\alpha I_{D C} s(t)$, where $\alpha \in[0,1]$ is the modulation index. To maintain linear currentto-light conversion and avoid clipping distortion, the LED transmitter has a constraint on its input power. Since the

\footnotetext{
${ }^{1}$ In this paper, we treat each LED transmitter as a single LED. However, an LED transmitter may be made up of a series of individual LEDs, called an LED fixture, in practice.
}

dynamic range of the LED is $I_{D C} \pm \alpha I_{D C}, x(t ; \alpha)$ is subject to the amplitude constraint $|x(t ; \alpha)| \leq \alpha I_{D C}$. Therefore, the optical power of the emitted signal from the LED can be described as $P_{T X}=\eta\left(I_{D C}+x(t ; \alpha)\right)$, where $\eta(W / A)$ is the current-to-light conversion efficiency. Therefore, the received signal voltage $y(t)$ after removing the $\mathrm{DC}$ bias is given by

$$
y(t)=h x(t ; \alpha)+w(t)
$$

where $h=\eta G B T$ is the channel gain, $G<1$ is the path gain, $B$ is the responsivity of a $\mathrm{PD}(A / W), T$ is the transimpedance amplifier gain $(V / A)$ and $w(t) \sim \mathcal{N}\left(0, \sigma^{2}\right)$ is a zero-mean additive white Gaussian noise (AWGN) term. $\sigma^{2}$ is given by [13]

$$
\sigma^{2}=\sigma_{\text {thermal }}^{2}+\sigma_{\text {shot }}^{2}+\left(P_{\text {ISI }} B\right)^{2}
$$

where $\sigma_{\text {thermal }}^{2}$ and $\sigma_{\text {shot }}^{2}$ denote variances of the thermal noise in the receiver electronic circuits, i.e., the amplifier noise, and the shot noise caused by ambient illumination from sunlight or other light sources, respectively. These two noise sources are well modeled as an additive white Gaussian process [13]. Also, $P_{\text {ISI }}$ denotes the equivalent optical power due to the intersymbol interference (ISI) caused by the multi-path reflection in a VLC channel. For high data rates (beyond $100 \mathrm{Mbps}$ ), the impact of the reflected lights on $\sigma^{2}$ can be significant due to the high ISI [13]. However, for low data rates (e.g., $10 \mathrm{Mbps}$ ), the reflected light can be beneficial since it contributes to increasing the received signal power whilst the ISI can be neglected by applying a simple transversal equalizer [14]. We assume a low data rate system enough to ignore ISI for the following analysis ${ }^{2}$.

According to [13], the Line-of-Sight (LoS) path gain can be described as

$$
G_{\mathrm{LoS}}=\left\{\begin{array}{ccc}
\frac{(m+1) A_{\mathrm{RX}}}{2 \pi l^{2}} \cos ^{m}(\phi) \cos (\psi) & \text { for } & |\psi| \leq \Psi_{\mathrm{FoV}} \\
0 & \text { for } & |\psi|>\Psi_{\mathrm{FoV}}
\end{array}\right.
$$

where $A_{R X}=A_{P D} K^{2} / \sin ^{2}\left(\Psi_{\mathrm{FOV}}\right)$ is the receiver collection area and $A_{P D}$ is the detector area of a PD. $\kappa$ is the refractive index of the optical concentrator at the receiver and $\Psi_{\mathrm{FOV}}$ is the received field of view of the PD. $m=-\ln (2) / \ln \left(\cos \phi_{1 / 2}\right)$ is the order of Lambertian emission with half illuminance at $\phi_{1 / 2}$. $l$ is the distance between the LED transmitter and receiver, and $\phi$ and $\psi$ are the irradiance and incidence angles at the LED transmitter and receiver, respectively.

The diffused path gain reflected by the walls was studied in [15]. The proposed approach calculated the reflection channel gain by partitioning a room into many elementary reflectors and summing up the impulse response contributions from different orders of bounces. However, in this paper, we consider only the first reflection since the channel gain of the higher reflection link is small enough to be neglected [13]. Thus, the channel gain of the diffused link considering only the first reflection can be described by (4) at the top of the next page. As shown in Fig. $1, W_{k}$ denotes the $k$ th side wall of a cuboid room and $\rho_{k}$ is its reflectivity. $l_{\left\{L, \mathrm{~d} W_{k}\right\}}$ and

\footnotetext{
${ }^{2}$ This assumption can be justified since a PLS technique may be a means to refresh the secret keys used in upper layers, rather than to exchange a large data stream; this application may not require a high data rate.
} 


$$
G_{\mathrm{REF}}=\sum_{k=1}^{4} \iint_{W_{k}} \rho_{k} \frac{(m+1)}{2 \pi l_{\left\{L, \mathrm{~d} W_{k}\right\}}^{2}} \cos ^{m}\left(\phi_{\left\{L, \mathrm{~d} W_{k}\right\}}\right) \cos \left(\psi_{\left\{L, \mathrm{~d} W_{k}\right\}}\right) \frac{A_{\mathrm{RX}}}{\pi l_{\left\{\mathrm{d} W_{k}, R\right\}}^{2}} \cos \left(\phi_{\left\{\mathrm{d} W_{k}, R\right\}}\right) \cos \left(\psi_{\left\{\mathrm{d} W_{k}, R\right\}}\right) F\left(\psi_{\left\{\mathrm{d} W_{k}, R\right\}}\right) \mathrm{d} W_{k}
$$

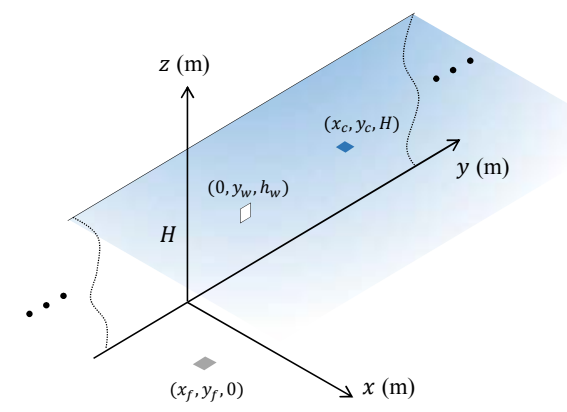

Fig. 2. Geometry of the infinite LED transmitter, wall and floor.

$l_{\left\{\mathrm{d} W_{k}, R\right\}}$ are the distances from the LED transmitter to the differential wall reflector $\mathrm{d} W_{k}$ and from $\mathrm{d} W_{k}$ to the receiver, respectively. $\phi_{\left\{L, \mathrm{~d} W_{k}\right\}}$ and $\psi_{\left\{L, \mathrm{~d} W_{k}\right\}}$ are the irradiance and incidence angles from the LED to $\mathrm{d} W_{k}$, respectively. Also, $\phi_{\left\{\mathrm{d} W_{k}, R\right\}}$ and $\psi_{\left\{\mathrm{d} W_{k}, R\right\}}$ are the irradiance and incidence angles from $\mathrm{d} W_{k}$ to the receiver, respectively. $F\left(\psi_{\left\{\mathrm{d} W_{k}, R\right\}}\right)$ is the indicator function defined by

$$
F\left(\psi_{\left\{\mathrm{d} W_{k}, R\right\}}\right)=\left\{\begin{array}{lll}
1 & \text { for } \quad\left|\psi_{\left\{\mathrm{d} W_{k}, R\right\}}\right| \leq \Psi_{\mathrm{FoV}} \\
0 & \text { for } \quad\left|\psi_{\left\{\mathrm{d} W_{k}, R\right\}}\right|>\Psi_{\mathrm{FoV}}
\end{array}\right.
$$

Therefore, the channel gain considering both the LoS and the diffused light can be described as in [13] by

$$
h=\eta\left(G_{\mathrm{LoS}}+G_{\mathrm{REF}}\right) B T .
$$

\section{Performance Metric}

For Gaussian VLC channels with amplitude constraints, the peak SNR at the receiver can be described as

$$
\gamma=\frac{\alpha^{2} I_{D C}^{2} h^{2}}{\sigma^{2}} .
$$

In addition, the SOP can be described by

$$
P_{S O}=\mathbb{P}\left(\gamma_{U E}<\max _{e \in \Phi_{E}} \gamma_{E D_{e}}\right) \stackrel{(a)}{=} 1-\exp \left(-\iint_{\mathcal{A}} \lambda_{E}(\mathbf{x}) \mathrm{d} \mathbf{x}\right)
$$

where $\gamma_{U E}$ and $\gamma_{E D_{e}}$ are the SNR of the UE and the ED $e \in \Phi_{E}$, respectively. For (a), we apply the void probability of PPP as in [11]. $\lambda_{E}(\mathbf{x})$ is the density of EDs at the point $\mathbf{x} \in \mathbb{W}^{2}$, where $\mathbb{W}^{2}$ is the set of points in the work plane of the room. Note that when the ED point process is homogeneous, $\lambda_{E}$ is a constant. Also, $\mathcal{A}=\left\{\mathbf{x} \mid \gamma(\mathbf{x})>\gamma_{U E}\right\}$, where $\gamma(\mathbf{x})$ denotes the received SNR at the point $\mathbf{x}$, i.e., $\gamma(\mathbf{x}): \mathbb{W}^{2} \rightarrow \mathbb{R}^{+}$.

\section{The Domain of Interest with Reflection}

In this section, we investigate how the reflection affects on the received SNR according to the receiver's distance from the wall. Looking at (4), the inverse-square law with respect to the distance implies that the middle bulk of the room will not be affected in SNR by reflections. Thus, we quantify a region of interest for which we should consider the reflection from the secrecy viewpoint by defining the generalized error in $S N R$ that occurs when reflections are ignored. For a theoretic investigation, we consider an infinitely large room where an infinite number of LEDs are attached to the ceiling, and the distances among LEDs are infinitesimal. Fig. 2 illustrates the geometry of the infinite room with the infinite number of LEDs (the shaded plane denotes LEDs). To deal with the infinite number of LEDs, we characterize the emitted optical power of LEDs by the power density per unit of LED area $P_{T}\left[\mathrm{~W} / \mathrm{m}^{2}\right]$.

First, the optical power density $P_{W}\left[W / \mathrm{m}^{2}\right]$ reaching from the LEDs to a wall reflector located at $\left(0, y_{w}, h_{w}\right)$ can be described by

$$
\begin{aligned}
P_{W} & =\int_{-\infty}^{\infty} \int_{0}^{\infty} P_{T} \frac{m+1}{2 \pi l_{\{T, W\}}^{2}}\left(\frac{H-h_{w}}{l_{\{T, W\}}}\right)^{m}\left(\frac{x_{c}}{l_{\{T, W\}}}\right) \mathrm{d} x_{c} \mathrm{~d} y_{c} \\
& \stackrel{(a)}{=} \frac{P_{T}}{2}
\end{aligned}
$$

where $l_{\{T, W\}}=\sqrt{x_{c}^{2}+\left(y_{c}-y_{w}\right)^{2}+\left(H-h_{w}\right)^{2}}$. For (a) and the following analysis, we assume all of the LED transmitters have the Lambertian emission pattern, i.e., $\phi_{1 / 2}=60^{\circ}(m=1)$. This result implies that regardless of the location of the wall reflector, $P_{W}$ is constant as a half of $P_{T}$. This is because the upper area of the wall is close to the ceiling so that it can receive strong signals from the LEDs, but the irradiance angle from the LED to the wall reflector quickly approaches to $90^{\circ}$ as the transmitter goes away from the wall. Thus, the number of transmitters that can meaningfully contribute to the received signal power of the upper area quickly decreases. In contrast, the lower area of the wall is relatively far from the ceiling, but the irradiance angle from the LED to the wall reflector does not increase as much as the upper area. Thus, the larger number of distant LEDs can contribute to the signal power received at the lower area wall. Therefore, on average, the received signal can be constant.

Then, the optical power density $P_{F, R E F}\left[\mathrm{~W} / \mathrm{m}^{2}\right]$ reaching to the point $\left(x_{f}, y_{f}, 0\right)$ on the floor after being reflected by the wall can be described by

$$
\begin{aligned}
P_{F, R E F} & \stackrel{(b)}{=} \int_{-\infty}^{\infty} \int_{0}^{H} \rho \frac{P_{T}}{2} \frac{1}{\pi l_{\{W, F\}}^{2}}\left(\frac{x_{f}}{l_{\{W, F\}}}\right)\left(\frac{h_{w}}{l_{\{W, F\}}}\right) \mathrm{d} h_{w} \mathrm{~d} y_{w} \\
& =\rho \frac{P_{T}}{2}\left(\frac{1}{2}-\frac{x_{f}}{2 \sqrt{H^{2}+x_{f}^{2}}}\right)
\end{aligned}
$$

where $\rho$ is the reflectivity of the wall and $l_{\{W, F\}}=$ $\sqrt{x_{f}^{2}+\left(y_{w}-y_{f}\right)^{2}+h_{w}^{2}}$. For (b) and the following analysis, we assume that a receiver's PD faces up perpendicular to the floor. This result verifies that as the receiver goes far from the wall, i.e., $x_{f} \rightarrow \infty$, its received power from the reflection approaches zero. The Taylor expansion of (10) at $x_{f} \rightarrow \infty$ is 


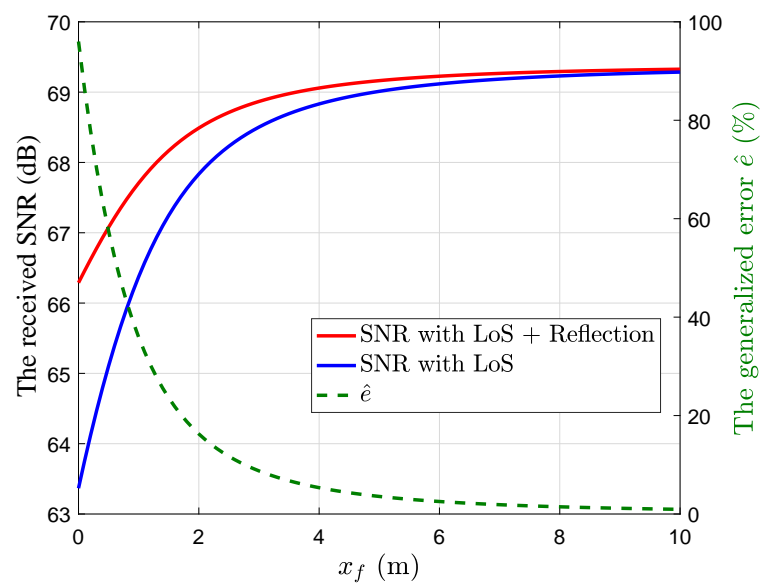

Fig. 3. SNR for different $x_{f}$ and the generalized error $\hat{e}$. $\rho=0.8$ (white wall) is applied.

$\rho P_{T} H^{2} /\left(8 x_{f}^{2}\right)+O\left(x_{f}^{-4}\right)$, which shows that the optical power density by the reflection decays very quickly as the PD moves away from the wall as we anticipated at the beginning of this section.

Finally, the received signal power density at $\left(x_{f}, y_{f}, 0\right)$ on the floor by the LoS component can be calculated by

$$
\begin{aligned}
P_{F, L o S} & =\int_{-\infty}^{\infty} \int_{0}^{\infty} P_{T} \frac{m+1}{2 \pi l_{\{T, F\}}^{2}}\left(\frac{H}{l_{\{T, F\}}}\right)^{m}\left(\frac{H}{l_{\{T, F\}}}\right) \mathrm{d} x_{c} \mathrm{~d} y_{c} \\
& =\frac{P_{T}}{2}\left(1+\frac{x_{f}}{\sqrt{H^{2}+x_{f}^{2}}}\right)
\end{aligned}
$$

where $l_{\{T, F\}}=\sqrt{\left(x_{c}-x_{f}\right)^{2}+\left(y_{c}-y_{f}\right)^{2}+H^{2}}$. Note that if the receiver is located very far from the wall, i.e., $x_{f} \rightarrow \infty$, the received power density at the receiver equals to $P_{T}$. The correction term here is $O\left(x_{f}^{-2}\right)$.

From (10) and (11), we can quantify the generalized error in the received $\mathrm{SNR}^{3}$ that occurs when reflections are ignored as

$$
\hat{e}=\frac{\left(P_{F, R E F}+P_{F, L o S}\right)^{2}-P_{F, L o S}^{2}}{P_{F, L o S}^{2}} \times 100(\%) .
$$

Fig. 3 shows the generalized error $\hat{e}$ as a function of the distance between the wall and the PD $x_{f}$, and also provides the received SNR considering LoS plus the 1st reflection as well as considering only the LoS component, respectively. It is shown that when the PD is located near to the wall, the contribution of the reflection to SNR is significant, while it drastically deceases as it moves away from the wall. Note $\hat{e}$ can be larger than $100 \%$ when $P_{F, L o S}$ is lower than $P_{F, R E F} /(\sqrt{2}-1)$.

\section{SOP WITH REFLECTIONS}

In this section, we investigate how reflection affects the SOP according to the location of the UE and the LED configuration when EDs are randomly distributed. As discussed in the previous section, since the reflection differently affects the

\footnotetext{
${ }^{3}$ Note that $P_{\mathrm{F}, \mathrm{REF}}$ and $P_{\mathrm{F}, \mathrm{LoS}}$ are the optical power densities, it is needed to square them to change to the electrical power.
}

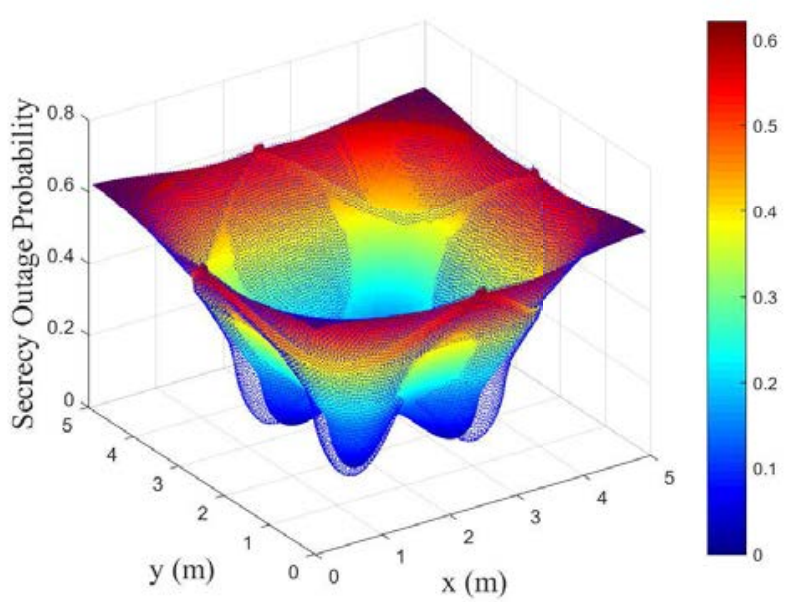

(a) SOP

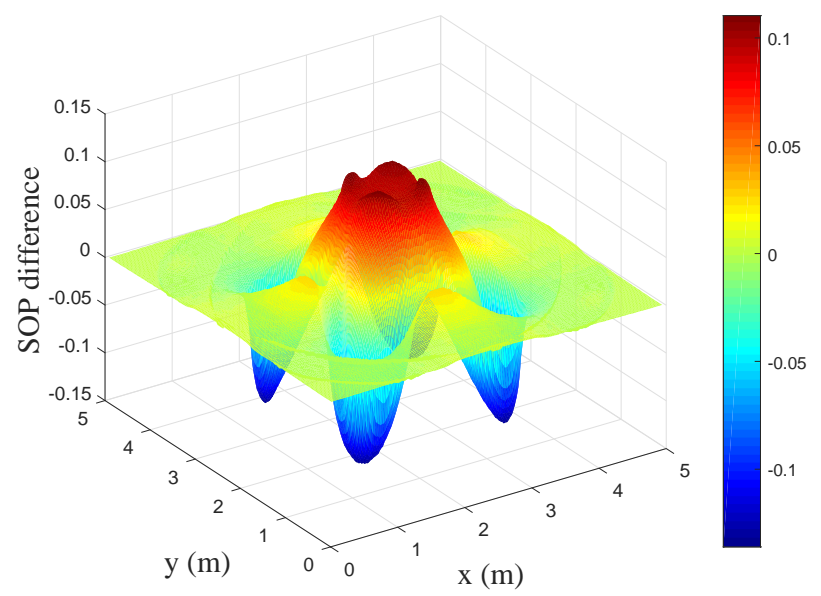

(b) The difference of SOP

Fig. 4. The SOP as a function of the UE location. The dotted blue surface in (a) is the SOP with LoS and reflection. The filled surface in (a) is the SOP only with LoS. (b) shows the SOP difference, i.e., the dotted surface minus the filled surface. The locations of four LEDs are $(2.5 \pm 1.2,2.5 \pm 1.2,3) . W=5 \mathrm{~m}, L=5 \mathrm{~m}, H=3 \mathrm{~m}$, and $H_{W}=0.8 \mathrm{~m}$ are used. $\lambda_{E}=0.04$ and $\rho=0.8$ are used.

received SNR according to the location of a receiver, we can anticipate the effect of the reflection on the SOP would also change according to the locations of the UE and EDs. In other words, if the UE takes advantage from the reflection more than randomly distributed EDs, the SOP would decrease by the reflection. To verify this prediction, Fig. 4 shows the numerical result of the SOP as a function of the location of the UE. We assume that four transmitters are attached to the ceiling at $(2.5 \pm 1.2,2.5 \pm 1.2,3)$ in the $5 \times 5 \times 3 \mathrm{~m}^{3}$ room. Also, $H_{w}=0.8 \mathrm{~m}, \rho=0.8$ and $\lambda_{E}=0.01$ are used. The filled surface in Fig. 4(a) shows the SOP when only the LoS component is considered, while the dotted surface considers the reflection as well. In addition, the SOP difference, i.e., the dotted surface minus the filled surface, is given in Fig. 4(b). Note that the positive SOP difference denotes a benefit for EDs. As we anticipate, the SOP increases, decreases or just remains unchanged as the location of the UE changes. 


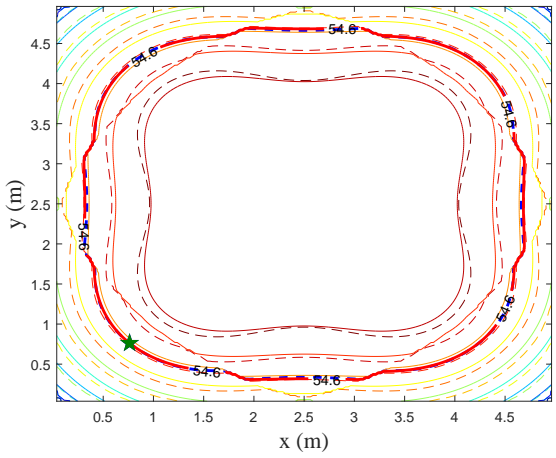

(a) The case of SOP remaining. The UE is located at $(0.76,0.76,0.8)$

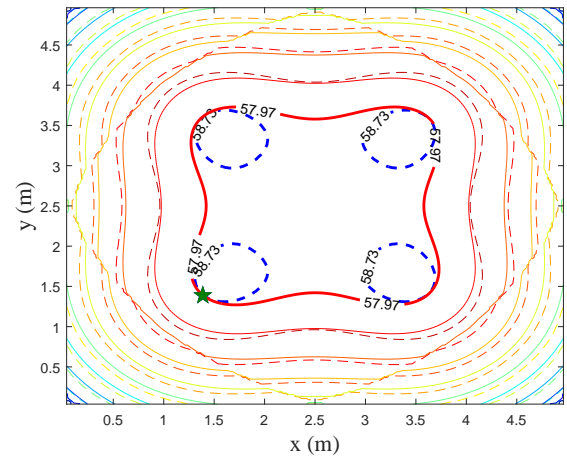

(b) The case of SOP decreasing. The UE is located at $(1.39,1.39,0.8)$.

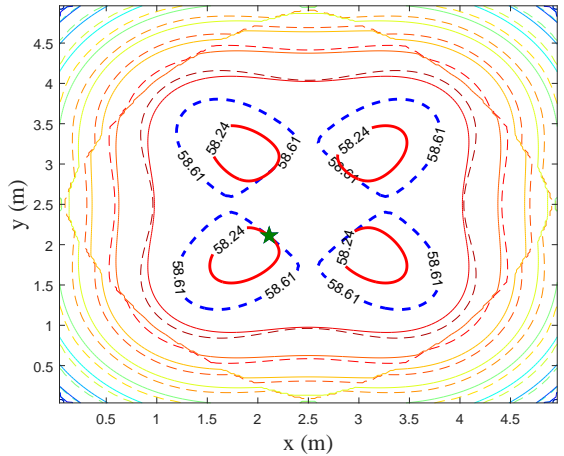

(c) The case of SOP increasing. The UE is located at $(2.11,2.11,0.8)$.

Fig. 5. The contour plot of the SNR. The green star is the location of the UE. The thick solid red line and the thick dashed blue line denote the possible locations of EDs causing the secrecy outage when the LoS component is considered and when the LoS and the reflection are considered, respectively. Four LED transmitters are located at $(2.5 \pm 1.2,2.5 \pm 1.2,3)$. The reflective index of walls $\rho=0.8$ is used.

Fig. 5 is the contour plot of the SNR as a function of a receiver's location explaining how the SOP changes according to the location of the UE. The solid contour lines are only for the LoS, and the dotted contour lines are for the combination of the reflection and the LoS. The green star is the location of the UE. Also, the thick solid red lines and the thick dashed blue lines denote the possible locations of EDs causing the secrecy outage when the LoS component is considered and when the LoS and the reflection are considered, respectively. In other words, the thick solid red lines and the thick dashed blue lines indicate the area in which the SNR is higher than that of the UE when the location of the UE is given as the green star. As can be seen in Fig. 5(a), when the UE is located at an outer area, i.e., $(0.76,0.76,0.8)$, the possible area of EDs causing the secrecy outage does not change by the reflection; the thick solid red and thick dashed blue lines are identical. Thus, from (8), the SOP does not change. On the other hand, when the UE is near to the transmitter, i.e., $(1.39,1.39,0.8)$, the possible area of EDs causing the secrecy outage is reduced by the reflection as shown in Fig. 5(b), which results in the decrease of the SOP. In contrast, when the UE is located in the center of the room, i.e., $(2.11,2.11,0.8)$, the possible area of EDs causing the secrecy outage broadens as shown in Fig. 5(c), then the SOP increases. This investigation verifies that the change of the SNR contour by the reflection also changes the possible area of EDs causing the secrecy outage; thus the SOP changes according to the location of the UE.

From this investigation, we can also note that if the received SNR is a quasiconcave function defined on the room work plane, there is no difference of the SOP between considering $\mathrm{LoS}$ and considering the combination of LoS and the reflection. This is because the reflection may change the absolute power of the received signal, but the reflection cannot change the SNR order over the entire room under the quasiconcavity condition. More specifically, the values of the received SNR $\gamma\left(\mathbf{x}_{1}\right)$ and $\gamma\left(\mathbf{x}_{2}\right)$ for $\mathbf{x}_{1}, \mathbf{x}_{\mathbf{2}} \in \mathbb{W}^{2}$ can change by the reflection, but $\left[\gamma\left(\mathbf{x}_{1}\right)>\gamma\left(\mathbf{x}_{\mathbf{2}}\right)\right]$ does not change as far as $\gamma(\mathbf{x})$ is quasiconcave, where $[\cdot]$ denotes the Iverson bracket. The received SNR function $\gamma(\mathbf{x})$ is quasiconcave if

$$
\gamma\left(\lambda \mathbf{x}_{1}+(1-\lambda) \mathbf{x}_{2}\right) \geq \min \left\{\gamma\left(\mathbf{x}_{1}\right), \gamma\left(\mathbf{x}_{2}\right)\right\}
$$

TABLE I. Simulation Parameters

\begin{tabular}{c|c}
\hline \multicolumn{2}{c}{ LED electrical and optical characteristics } \\
\hline Average optical power per LED & $4 \mathrm{~W}$ \\
Optical power / current $\eta$ & 1 \\
Nominal half-intensity angle $\Phi_{1 / 2}$ & $60^{\circ}$ \\
Modulation index $\alpha$ & 0.5 \\
\hline \multicolumn{2}{c}{ Optical receiver characteristics } \\
\hline Field of View $\Psi_{\text {FoV }}$ & $90^{\circ}$ \\
Lens refractive index $\kappa$ & 1.5 \\
Photodetector's responsivity & $0.54 \mathrm{~mA} / \mathrm{mW}$ \\
Photodetector's physical area $A_{P D}$ & $1 \mathrm{~cm}{ }^{2}$ \\
Transimpedence amplifier gain T & $1 \mathrm{mV} / \mathrm{mA}$ \\
Noise power $\sigma^{2}$ & $-117 \mathrm{dBm}$ \\
\hline
\end{tabular}

for all $\mathbf{x}_{1}, \mathbf{x}_{2} \in \mathbb{W}^{2}$ and $\lambda \in[0,1]$ [16]. Note that, in practice, whether the SNR function is quasiconcave or not depends on the LED configuration, such as LED arrangement, transmitted optical power of each LED, LED emission pattern and so on.

\section{Simulation Results}

In this section, we provide simulation results to verify our analysis. The simulation parameters for the transmitter and receiver are provided in Table I.

Fig. 6 shows the maximum and minimum SOP differences for different intervals between transmitters $d$, i.e., we assume four LED transmitters and set the locations of transmitters as $(W / 2 \pm d, L / 2 \pm d, 3)$. In other words, as shown in Fig. 4(b), we find the UE location that yields the maximum SOP difference and that becomes the data point on the blue curve for a given $d$. Also, the minimum point becomes the data point on the red curve. The blue curve denotes the reflection benefits for EDs, and the red curve denotes the reflection benefits for the UE. As shown in the figure, the SOP difference before $d^{*}$ is almost zero, while it dramatically increases after that point. $d^{*}$ denotes the maximum value of $d$ satisfying (13). To simplify the calculation of $d^{*}$, we ignore the reflection component in (6) because the reflection component mainly affects the outer area of the room as we discussed in Section IV, while the SNR fluctuation violating the quasiconcavity exists at the inner area of the room. Therefore, this result verifies that we can eliminate the effect of the reflection on the SOP by adjusting 


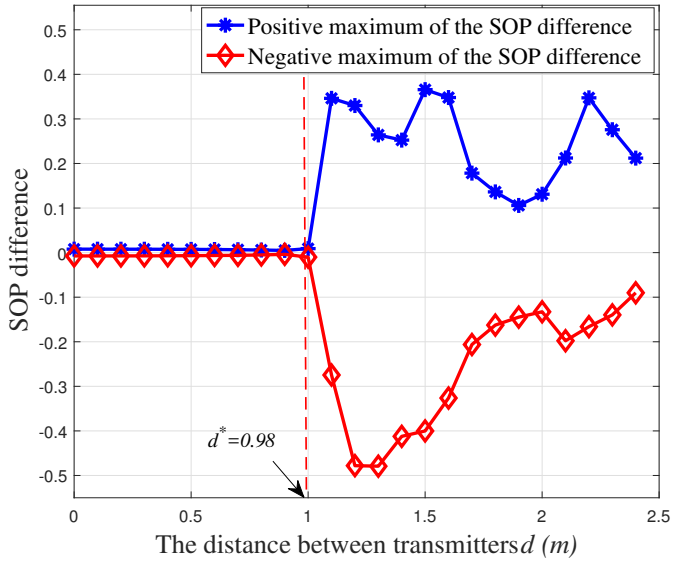

Fig. 6. The maximum and minimum differences of the SOP. Four LED transmitters are located at $(2.5 \pm d, 2.5 \pm d, 3) . \lambda_{E}=0.1, W=$ $5 \mathrm{~m}, L=5 \mathrm{~m}, H=3 \mathrm{~m}$, and $H_{W}=0.8 \mathrm{~m}$ are used. The reflective index of walls $\rho=0.8$ is used.

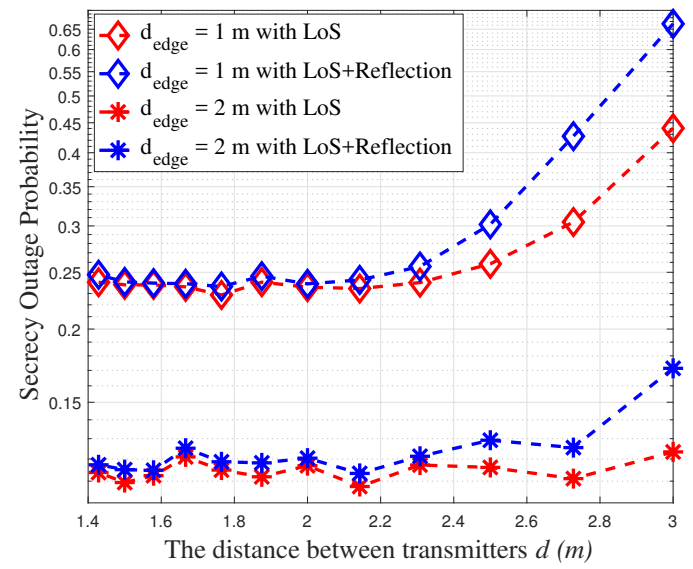

Fig. 7. The SOP for different distances between LED transmitters. $N \times N$ LED transmitters are uniformly located in the $L \times W \mathrm{~m}^{2}$ room. Thus the horizontal and vertical distances between transmitters are defined by $L / N$ and $W / N$, respectively. $\lambda_{E}=0.5, W=30 \mathrm{~m}$, $L=30 \mathrm{~m}, H=3 \mathrm{~m}, H_{W}=0.8 \mathrm{~m}$, and $\rho=0.8$ are used.

the arrangement of LED transmitters and making the SNR function satisfy (13).

Fig. 7 shows the SOP when the EDs are distributed in a very large $30 \times 30 \mathrm{~m}^{2}$ room. To investigate the impact of the reflection with respect to the distance between the wall and EDs, we assume that EDs intentionally try to be located in the edge area, i.e., within $d_{\text {edge }}$ from the wall, in order to escape the vigilance of the UE. In contrast, we assume that one UE is randomly located anywhere in the room, which can be modeled by a Binomial point process (BPP) with a single point. In addition, to change the distance between the transmitters $d$, we increase or decrease the number of the transmitters given the room size. Similarly to Fig. 6, we can note that the SOP difference between considering LoS and considering the conbination of LoS and the reflection increases as the interval of transmitter increases. Moreover, when EDs are located near to the wall, the SOP difference also increases. Specifically, for $d=3 \mathrm{~m}$, the SOP difference is 0.223 for $d_{\text {edge }}=1 \mathrm{~m}$ and 0.048 for $d_{\text {edge }}=2 \mathrm{~m}$, respectively. This result verifies that EDs which are located nearer to the wall can take a higher advantage of the reflection, which results in the higher increase of the SOP.

\section{CONCLUSION}

In this paper, we studied the impact of the multipath reflection on the SOP when EDs are randomly distributed. We quantified the domain of interest by defining the generalized error in SNR that occurs when reflections are ignored. The simulation results verified that EDs being located nearer to the wall could take a higher advantage from the reflection, which results in a higher SOP. We also showed that the impact of the reflection depends on the location of the UE and the configuration of LEDs. The effect of the reflection on the SOP could be removed by adjusting the LED arrangement and making the SNR function quasiconcave. Our results provide useful insight that can be used to enhance the secrecy in VLC systems.

\section{ACKNOWLEDGMENT}

The work was supported in part by EPSRC grant number EP/N002350/1 ("Spatially Embedded Networks").

\section{REFERENCES}

[1] D. Karunatilaka, F. Zafar, V. Kalavally, and R. Parthiban, "LED based indoor visible light communications: State of the art," IEEE Commun. Surveys \& Tutorials, vol. 17, no. 3, pp. 1649-1678, 2015.

[2] M. Shafi, A. F. Molisch, P. J. Smith, T. Haustein, P. Zhu, P. De Silva, F. Tufvesson, A. Benjebbour, and G. Wunder, "5G: A tutorial overview of standards, trials, challenges, deployment and practice," IEEE Journal on Selected Areas in Commun., vol. 8716, no. c, pp. 1-1, 2017.

[3] H. Haas, L. Yin, Y. Wang, and C. Chen, "What is LiFi?" Journal of Lightwave Technology, vol. 34, no. 6, pp. 1533-1544, Mar. 2016.

[4] M. Bloch and J. Barros, Physical-Layer Security: From Information Theory to Security Engineering. Cambridge University Press, 2011.

[5] Y. Liu, H. H. Chen, and L. Wang, "Physical layer security for next generation wireless networks: Theories, technologies, and challenges," IEEE Commun. Surveys Tutorials, vol. 19, no. 1, pp. 347-376, Q1 2017.

[6] A. Mostafa and L. Lampe, "Securing visible light communications via friendly jamming," in IEEE Globecom Workshops in Austin, USA, Dec. 2014.

[7] —_ "Physical-layer security for indoor visible light communications," in IEEE ICC in Sydney, Australia, Jun. 2014.

[8] — "Physical-layer security for MISO visible light communication channels," IEEE Journal on Selected Areas in Commun., vol. 33, no. 9, pp. 1806-1818, Sep. 2015.

[9] H. Zaid, Z. Rezki, A. Chaaban, and M. S. Alouini, "Improved achievable secrecy rate of visible light communication with cooperative jamming," in IEEE GlobalSIP in Orlando, USA, Dec. 2015.

[10] M. A. Arfaoui, Z. Rezki, A. Ghrayeb, and M. S. Alouini, "On the secrecy capacity of MISO visible light communication channels," in IEEE Globecom in Washington D.C., USA, Dec. 2016.

[11] S. Cho, G. Chen, and J. P. Coon, "Secrecy analysis in visible light communication systems with randomly located eavesdroppers," in 2017 IEEE ICC Workshops in Paris, France, May 2017.

[12] J. Classen, J. Chen, D. Steinmetzer, M. Hollick, and E. Knightly, "The spy next door: Eavesdropping on high throughput visible light communications," in the 2nd ACM Int. Workshop on VLC Systems in Paris, France. ACM, 2015.

[13] T. Komine and M. Nakagawa, "Fundamental analysis for visible-light communication system using LED lights," IEEE Trans. on Consumer Electronics, vol. 50, no. 1, pp. 100-107, Feb 2004.

[14] S. Randel, F. Breyer, S. C. J. Lee, and J. W. Walewski, "Advanced modulation schemes for short-range optical communications," IEEE Journal of Selected Topics in Quantum Electronics, vol. 16, no. 5, pp. 1280-1289, Sep. 2010.

[15] J. M. Kahn and J. R. Barry, "Wireless infrared communications," Proceedings of the IEEE, vol. 85, no. 2, pp. 265-298, Feb 1997.

[16] S. Boyd and L. Vandenberghe, Convex Optimization. New York, NY, USA: Cambridge University Press, 2004. 